\title{
Comparison of Wet Mount Examination with Giemsa Staining and Fluorescent Staining for Detection of Trichomonas vaginalis in Clinically Suspected Cases of Vulvovaginitis
}

\author{
Vartika Paliwal $^{1}$, Arti Jain ${ }^{1}$, Avinash Laghawe ${ }^{1}$, Kaore Navinchandra ${ }^{2}$ \\ and Tukaram Prabhu ${ }^{1}$ \\ ${ }^{1}$ People's College of Medical Sciences \& Research Center, Bhanpur Road, \\ Bhopal -462037 Madhya Pradesh, India \\ ${ }^{2}$ Raipur Institute of Medical Sciences, Gram Godhi, Bhansoj Road, Raipur, (C.G), India \\ *Corresponding author
}

\section{A B S T R A C T}

\section{Keywords}

Trichomonas vaginalis, Wet mount examination, Giemsa staining, Acridine orange staining.

Article Info

Accepted:

15 February 2017

Available Online

10 March 2017
Trichomonas vaginalis is an important cause of the premature rupture of membranes, premature delivery, pelvic inflammatory disease, urethritis, and chronic prostatitis. Wet mount is the most widely used method for the detection of $T$. vaginalis in clinically suspected cases of vulvovaginitis. The purpose of this Study was to compare various microscopic techniques for detection of $T$. vaginalis like wet mount examination, Giemsa and Acridine orange staining. The Cross-sectional Analytical study was conducted in the Department of Microbiology, attached to a Tertiary Care Hospital in Central India after obtaining ethical clearance over a time period of two months. Out of 30 cases, only two $(6.66 \%)$ were detected positive for Trichomoniasis. All the 30 vaginal samples (cases) tested for detection of $T$. vaginalis were found to be negative by Wet mount examination. Whereas, Giemsa staining and Acridine orange staining were positive in $1(3.33 \%)$ and 2 $(6.66 \%)$ case respectively. Present study highlights that Acridine orange staining was found to be the best microscopic method when compared with wet mount examination and Giemsa staining. This will also provide rapid screening of vaginal smears in patients suffering from vaginal discharge and thus, help in early diagnosis and prompt treatment of patients.

\section{Introduction}

Trichomoniasis is the most common non-viral sexually transmitted disease (STD) worldwide. It was estimated that approx. 7.4 million new cases of Trichomonas vaginalis (T. vaginalis) infection occurred in 2000 in USA (Weinstock et al., 2004).

Population-level data from the National Longitudinal Study of Adolescent Health have documented that $T$. vaginalis affects
$2.8 \%$ of young adult women, a prevalence seven times higher than that of Neisseria gonorrhoeae in this age group (Miller et al., 2005).

Vaginitis due to Trichomonas vaginalis clinically manifests with symptoms of vaginal itching, odor, purulent, foul-smelling and vaginal discharge, which is associated with pruritis, dysuria and dyspareunia (Schwebke 
et al., 2004; Swygard et al., 2004). The discharge usually exceeds $\mathrm{pH}$ 6.0, and a 'strawberry' cervix, which is characterized by punctate hemorrhagic lesions, is described. These classic symptoms are only seen $20 \%$ of the time, which often leads to women with these vaginal symptoms being empirically treated for yeast infections or bacterial vaginosis.

Recent studies also show that $T$. vaginalis is an important cause of the premature rupture of membranes, premature delivery, pelvic inflammatory disease, urethritis, and chronic prostatitis.

Trichomoniasis infection also enhances the transmission of human immunodeficiency virus infection (Nanda et al., 2006). T.vaginalis is highly prevalent and is associated with adverse outcome, improved detection of $T$. vaginalis is needed.

In various clinical settings, wet mount is the most widely used method for the detection of $T$. vaginalis in clinically suspected cases of vulvovaginitis (Wiese et al., 2000).

Although, wet mount examination is most cost effective diagnosis test, but it has low sensitivity resulting in under diagnosis of the diseases. Therefore, to overcome this problem, various staining techniques like Giemsa and Acridine orange can be used along with wet mount examination for diagnosis of T.vaginalis infection.

Therefore the plan was to compare various microscopic techniques for detection of $T$. vaginalis like wet mount examination, Giemsa and Acridine orange staining.

\section{Materials and Methods}

This Cross-sectional Analytical study was conducted in the department of Microbiology, attached to a Tertiary care hospital in central
India over a time period of two months from $1^{\text {st }}$ August to $31^{\text {st }}$ October 2016 after obtaining Ethics Clearance.

A total of 30 vaginal swabs were collected from patient of reproductive age group (15-45 years).

\section{Collection of Samples}

A total of 30 vaginal swabs were collected from patient of reproductive age group (15-45 years) by clinicians in suspected cases of vulvovaginitis after written informed consent.

The clinicians obtained three vaginal samples/swabs [Himedia Laboratories Pvt. Limited, Mumbai] (first swab for detection of motile trichomonas by wet-mount microscopy $\&$ second swab was used to prepare smear for Giemsa staining and third swab was used to prepared smear for Acridine orange staining for detection of Trichomonas vaginalis) from each patient by swabbing the vaginal vault with the help of vaginal speculum and then send to laboratory within $10 \mathrm{~min}$ of collection.

The clinicians were asked to vary the order in which they obtained the vaginal swabs to prevent sampling bias.

\section{Procedure for sampling}

After the receiving of sample in the laboratory and procedure as followed-

$I^{\text {st }}$ swab - was placed in screw capped sterile plastic tube containing $0.5 \mathrm{ml}$ of $0.9 \%$ saline and used immediately for detection of motile Trichomonas by wet-mount microscopy.

\section{Wet Mount Examination}

First swab was processed for wet-mount microscopy. After vigorous mixing of the swab in the saline, the swab was pressed 
against the side of the tube to express as much fluid as possible. One drop of expressed fluid was placed on a clean glass slide with a cover slip and the slide was examined for detection of motile Trichomonas (exhibiting twisting motility) with a brightfield microscope at10x and $40 \mathrm{x}$ by the clinician or laboratory technician within 10 min of collection.

The Trichomonas were identified by their size $(10-20 \mu \mathrm{m})$, round or oval shape, and characteristic twitching motility (Cheesbrough, 2000).

II $^{\text {nd }}$ swab was used to prepare smear for Giemsa staining (Hi Media Laboratories, India) for detection of Trichomonas vaginalis. Procedure was as follows:

\section{Giemsa Staining}

The prepared smear was fixed by immersion in methanol for one minute and allowed to dry. It was then stained with Giemsa stain (Hi Media Laboratories, India), diluted 1 part to 19 parts of $1 / 15 \mathrm{M}$ phosphate buffer, $\mathrm{pH} 7.2$ for $10 \mathrm{~min}$ and scanned for Trichomonas vaginalis at $10 \mathrm{X}$ magnification and then at a magnification of 40x. At least 30 fields were examined before a negative finding was recorded. ${ }^{18}$

Both the internal and external structures of the organism were clearly visualized. The former stained dark blue with a red nucleus and the latter was sharply outlined, showing clearly the flagella and the undulating membrane [Figure A]. IIIrd swab was used to prepare smear for Acridine orange staining using staining kit by HiMedia, India for detection of Trichomonas vaginalis. Procedure was as follows:

\section{Acridine Orange Staining-}

The prepared smear was fixed with methanol for 2-3 min and covered with phenolic auramine dye [S042] which was allowed the stain to remain on the surface of the slide for 15 minutes without drying rinse with distilled water and draining off excess liquid. Slides were flooded with decolarizer [S043] for 2-3 mins. The slides were rinsed thoroughly with distilled water, and counterstained with [S044] for 3-4 mins by flooding. After being rinsed with distilled water $\&$ allowed to air dry, the slide was examined under a fluorescence microscope (with a 470-490 nm filter) at a magnification of 40X (Schee et al., 1999).

Epithelial cells fluoresced light green with a bright green nucleus. The nuclei of leucocytes (Pus cells) fluoresced bright green and bacteria stained bright red colour. Trophozoites of $T$. vaginalis were seen as characteristic brick red colour with a yellowish green nucleus. The images from the fluorescent microscopes cannot be captured as we don't have the facility for the same.

All data was maintained in Microsoft office Excel. Statistical analysis was carried out using Excel and test of proportion were used for analysis.

\section{Results and Discussion}

A total of 30 vaginal swabs were collected from patient of reproductive age group (15-45 years) after Institutional Ethics committee clearance.

The age wise distribution of the patients from whom the samples were collected is as given in Table 1.The observation for wet mount, Giemsa stain and Acridine orange stain for the samples collected were as given in Table 2 .

Of 30 cases only two (6.66\%) were detected positive for Trichomoniasis by Direct microscopic examination. 
Wet mount examination is the most frequently used method for diagnosis of Trichomoniasis in women. In study done by Razia Khatoon, Noor Jahan et al., (Razia et al., 2014) shows, out of the 615 cases, 25 (4.1\%) cases were positive for T. vaginalis infection by wet mount examination with sensitivity of $67.6 \%$. In our study, all the 30 cases were found to be negative by wet mount examination technique which may be because of chance as the sample size is small. The less number of organisms and inappropriate amount of sample collected due to variable absorption rate of the cotton swab used may lead to negative results. Our tertiary hospital being in the rural area and population with low socioeconomic category, mostly resort to the quacks and the over the counter prescriptions are very common. Majority of the patients reports to have taken anti protozoal treatments for protozoal infections very common in rural areas. Although, positive wet mount is diagnostic, a negative test cannot exclude Trichomoniasis because of low sensitivity.

Table.1 Age wise distribution of patients clinically suspected of Vulvovaginitis included in the study for detection of Trichomonas vaginalis

\begin{tabular}{|c|c|c|c|c|}
\hline \multirow{2}{*}{ Females } & 15 to 25 years & 26 to 35 years & 36 to 45 years & \\
\cline { 2 - 5 } & 8 & 12 & 10 & 30 \\
\hline
\end{tabular}

Table 2: Detection of T.vaginalis using Wet mount, Giemsa stain and Acridine orange stain

\begin{tabular}{|c|c|c|}
\hline Staining techniques performed & Positive cases(\%) & Negative cases $(\%)$ \\
\hline Wet Mount $(\mathrm{n}=30)$ & Nil $(0 \%)$ & $30(100)$ \\
\hline Giemsa staining $(\mathrm{n}=30)$ & $1(3.33)$ & $29(96.6)$ \\
\hline Acridine Orange staining $(\mathrm{n}=30)$ & $2(6.66)$ & $28(93.3)$ \\
\hline
\end{tabular}

Fig.1 Giemsa staining showing Trichomonas vaginalis

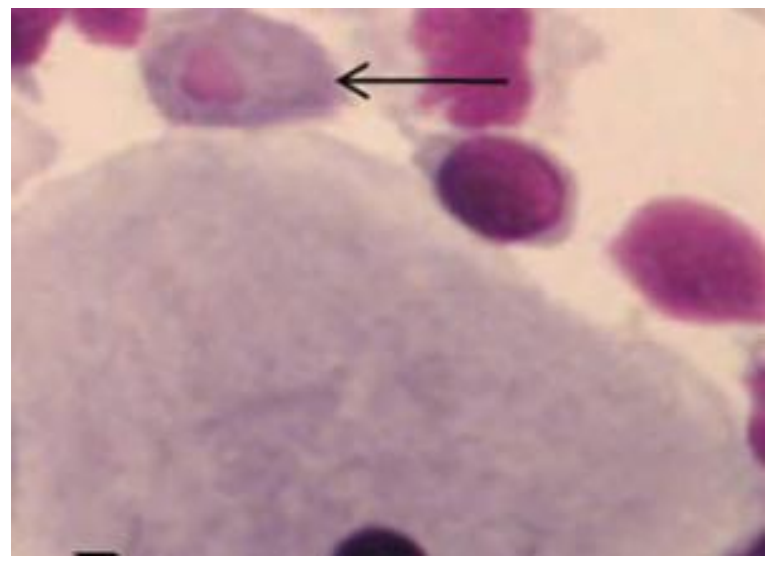

Study done by Razia Khatoon, NoorJahan et al., (2014) shows, the performance of Giemsa staining was good and it was able to detect
Trichomonas vaginalis in $30(4.9 \%)$ out of 615 patients. Studies done by different workers have reported varying range of 
sensitivity $(41-56 \%)$ of Giemsa stained smears for the diagnosis of Trichomonas vaginalis infection. In set up lacking immediate microscopic facilities Giemsa staining is very useful, where duly prepared and fixed smears can be transported to the laboratory for diagnosis. Also, with large number of patients attending gynaecology outpatient department, an immediate examination of a vaginal swab is virtually impossible. Unlike, wet mount examination, delay in transport has no significant impact on its reliability for diagnosing Trichomonas vaginalis. However, it is time consuming and needs technical expertise.

In our study, out of 30 cases examined, 2 cases were positive by Acridine orange staining. In similar study done by Razia Katoon, Noor Jahan et al., (2014), Acridine orange staining was positive in $37(6.0 \%)$ out of 615 patients, with sensitivity and specificity of $100 \%$ and $98.0 \%$ respectively. Whereas, in one study Acridine orange staining showed a high sensitivity and specificity of $71.43 \%$ and $99.44 \%$ respectively, in another study its sensitivity was only $47.5 \%$ in comparison to culture (Radonjic et al., 2006). Also, it has been reported that the sensitivity of Acridine orange staining is high $(67 \%)$ in women with T.vaginalis infection alone, and low (53\%) in women with multiple infections (Bickley et al., 1989).

In the present study, maximum number of cases of vaginal trichomoniasis was detected by Acridine orange staining. This is in agreement with a previously done study which showed that the infection rate detected by Acridine orange staining was higher than either culture or other vaginal swab examinations.

Although Acridine orange staining is easy to perform and rapid, its major disadvantage is that it requires special microscopic facilities, trained personnel and availability of the dye. Also, the smears lose their fluorescence with the passage of time, so permanent record is not possible. However, the rapidity, ease and reliability of Acridine orange staining justify its use in routine laboratory diagnosis of Trichomonal infection.

In conclusion, although wet mount examination is the most commonly used test in routine diagnosis of Trichomonas vaginalis infection, but staining techniques should be used as an additional diagnostic test in order to diagnose cases missed either due to unavailability of immediate microscopic facility or delay in the transport of samples to the laboratory for culture. Acridine orange staining was found to be the best microscopic method when compared with wet mount examination and Giemsa staining. Hence, it should be used in routine diagnosis of Trichomonas vaginalis infection in places where fluorescent microscopic facility is available. This will also provide rapid screening of vaginal smears in patients suffering from vaginal discharge and thus, help in early diagnosis and prompt treatment of patients. As Giemsa staining performed well in comparison to wet mount examination and can easily be done in routine, it should be used as an adjunct to wet mount microscopy in small setups lacking facilities for fluorescent microscopy. This will ultimately prove beneficial to patients in reducing morbidity and associated adverse health outcomes in the form of pelvic inflammatory diseases and increased risk of acquiring other sexually transmitted diseases including human immunodeficiency virus infection

Acknowledgement: We acknowledege the Indian Council of Medical Research for choosing the important and relevent topic for short term studentship program. 


\section{References}

Agarwal, S., Sharma, V., Sarin, R. 2005. Reproductive tract infections in womenPrevalence, HIVseropositivity and role of conventional methods in diagnosis. Indian J. Sex Transm. Dis., 26(2): 7377.

Bickley, L.S., Krisher, K.K., Punsalang, A. Jr, Trupei, M.A., Reichman, R.C., Menegus, M.A. 1989. Comparison of direct fluorescent antibody, Acridine orange, wet mount, and culture for detection of Trichomonas vaginalis in women attending a public sexually transmitted diseases clinic. Sex Transm. Dis., 16(3): 127-31.

Caliendo. A.M., Jordan. J.A., Green. A.M., Ingersoll. J., Diclemente, R.J. and Wingood G.M. 2005. Real- time PCR improves detection of Trichomonas vaginalis infection compared with culture using self- collected vaginal swabs. Infect. Dis. Obstetrics and Gynecol., 13(3): 145-150.

Cheesbrough, M. 2000. District Laboratory Practice in Tropical Countries, Part 2, Cambridge University Press.

Cherpes, T.L., Wiesenfeld, H.C., Melan, M.A., Kant, J.A. and Cosentino, L.A. 2006. The associations between pelvic inflammator diseases, Trichomonas vaginalis infection and positive Herpes simplex virus type 2serology. Sex Transm. Dis., 33: 747-752.

Fernando, S.D., Herath, S., Rodrigo, C., Rajapakse, S. 2011. Improving diagnosis of Trichomonas vaginalis infection in resource limited health care settings in Sri Lanka. J. Glob. Infect. Dis., 3(4): 324-28.

Hobbs, M.M., D.M. Lapple, L.F. Lawing, J.R. Schwebke, M.S. Cohen, H. Swygard, J. Atashili, P.A. Leone, W.C. Miller, and A.C. Sena. 2006. Methods for detection of Trichomonas vaginalis in the male partners of infected women: implications for control of trichomoniasis. J. Clin. Microbiol., 44: 3994-3999.

Johnston, V.J., Mabey, D.C. 2008. Global epidemiology and control of Trichomonas vaginalis. Curr. Opin. Infect. Dis., 21: 56-64.

Martens, G.M., Faro, S., Soper, D. 2001. Infectious disease in women. 1st edition. Philadelphia: Saunders.

Mason, P.R., Super, H., Fripp, P.J. 1976. Comparison of four techniques for the routine diagnosis of Trichomonas vaginalis infection. J. Clin. Path., 29: 154-57.

Miller, W.C., H. Swygard, M.M. Hobbs, et al. 2005. The prevalence of trichomoniasis in young adults in the United States. Sex. Transm. Dis., 32: 593-598.

Nanda, N., R.G. Michel, G. Kurdgelashvili, and K.A. Wendel. 2006. Trichomoniasis and its treatment. Expert Rev. Anti. Infect. Ther., 4: 125-135.

Pastorek, J.G., Cotch, M.F., Martin, D.H., et al. 1996. Clinical and microbiological correlates of vaginal trichomoniasis during pregnancy. The Vaginal Infections and Prematurity Study Group. Clin. Infect. Dis., 23: 1075-80.

Patil, M.J., Nagamoti, J.M., Metgud, S.C. 2012. Diagnosis of Trichomonas vaginalis from vaginal specimens by wet mount microscopy, in pouch TV culture system, and PCR. J. Glob. Infect. Dis., 4(1): 22-25.

Petrin, D., Delgaty, K., Bhatt, R., Garber, G. 1998. Clinical and microbiologicalaspects of Trichomonas vaginalis. Clin. Microbiol. Rev., 11: 300-17.

Radonjic, I.V., Dzamic, A.M., Mitrovic, S.M., Arsenijevic, V.S.A., Popadic, D.M., Zec, I.F.K. 2006. Diagnosis of Trichomonas vaginalis infection: The sensitivities and specificities of 
microscopy, culture and PCR assay. Eur. J. Obstet. Gynecol. Repro Biol., 126(1): 116-20.

Razia Katoon, Noor Jahan, et al. 2014. Evaluation of Different Staining Techniques in the Diagnosis of Trichomonas vaginalis Infection in Females of Reproductive Age Group. $J C D R$, vol. 8(12).

Schee, C.V.D., Belkum, A.V., Zwijgers, L., Brugge, E.V.D., O’Neill, E.L., Luijendijk, A.D., et al. Improved Diagnosis of Trichomonas vaginalis Infection by PCR Using Vaginal Swabs and Urine Specimens Compared to Diagnosis by Wet Mount Microscopy, Culture, and Fluorescent Staining. $J$. Clin. Microbiol., 37(12): 4127-30.

Schwebke, J.R., and D. Burgess. 2004. Trichomoniasis. Clin. Microbiol. Rev., 17: 794-803.

Swygard, H., A.C. Sena, M.M. Hobbs, and M.S. Cohen. 2004. Trichomoniasis: clinical manifestations, diagnosis and management. Sex Transm. Infect., 80: 91-95.
Van Der Pol, B., Kwok, C., Pierre-Louis, B., et al. 2008. Trichomonas vaginalis infection and human immunodeficiency virus acquisition in African women. $J$. Infect. Dis., 197: 548-54.

Weinstock, H., S. Berman, and W. Cates, Jr. 2004. Sexually transmitted diseases among American youth: incidence and prevalence estimates. Perspect. Sex. Reprod. Health, 36: 6-10.

Wiese, W., S.R. Patel, S.C. Patel, C.A. Ohl, and C.A. Estrada. 2000. A metaanalysis of the Papanicolaou smear and wet mount for the diagnosis of vaginal trichomoniasis. Am. J. Med., 108: 301308.

World Health Organization (WHO). 2005. Prevalence and incidence of selected sexually transmitted infections: Chlamydia trachomatis, Neisseria gonorrhoeae, syphilis, and Trichomonas vaginalis. Geneva, Switzerland: WHO, http://whqlibdoc.who.int/ publications/2011/9789241502450.

\section{How to cite this article:}

Vartika Paliwal, Arti Jain, Avinash Laghawe, Kaore Navinchandra and Tukaram Prabhu. 2017. Comparison of Wet Mount Examination with Giemsa Staining and Fluorescent Staining for Detection of Trichomonas vaginalis in Clinically Suspected Cases of Vulvovaginitis. Int.J.Curr.Microbiol.App.Sci. 6(3): 718-724. doi: https://doi.org/10.20546/ijcmas.2017.603.083 